\title{
BMJ Open Counselling for physical activity, life- space mobility and falls prevention in old age (COSMOS): protocol of a randomised controlled trial
}

\author{
Johanna Edgren, ${ }^{1,2}$ Saija Karinkanta, ${ }^{3}$ Taina Rantanen, ${ }^{1,2}$ Robin Daly, ${ }^{4}$ \\ Urho M Kujala, ${ }^{1}$ Timo Törmäkangas, ${ }^{1,2}$ Harri Sievänen, ${ }^{3}$ Pekka Kannus, ${ }^{5}$ \\ Ari Heinonen, ${ }^{1}$ Sarianna Sipilä, ${ }^{1,2}$ Lasse Kannas, ${ }^{1}$ Timo Rantalainen, ${ }^{1,2}$ \\ Outi Teittinen, ${ }^{6,7}$ R Nikander ${ }^{1,2,8}$
}

To cite: Edgren J, Karinkanta S, Rantanen T, et al. Counselling for physical activity, life-space mobility and falls prevention in old age (COSMOS): protocol of a randomised controlled trial. BMJ Open 2019:9:e029682. doi:10.1136/ bmjopen-2019-029682

- Prepublication history and additional material for this paper are available online. To view please visit the journal (http:// dx.doi.org/10.1136/bmjopen2019-029682).

Received 05 February 2019 Revised 24 July 2019 Accepted 19 August 2019
Check for updates

(C) Author(s) (or their employer(s)) 2019. Re-use permitted under CC BY-NC. No commercial re-use. See rights and permissions. Published by BMJ.

For numbered affiliations see end of article.

Correspondence to Dr Johanna Edgren; johanna.edgren@jyu.fi

\section{ABSTRACT}

Introduction The most promising way to promote active life years in old age is to promote regular participation in physical activity (PA). Maintaining lower extremity muscle function with good balance has been associated with fewer falls and the need of help from others. This article describes the design and intervention of a randomised controlled trial (RCT) investigating the effectiveness of a health and PA counselling programme on life-space mobility and falls rates in community-dwelling older adults at the Health Kiosk and/or Service Centre.

Methods and analysis Community-dwelling men and women $(n=450)$ aged 65 years and over with early phase mobility limitation will be recruited to a 24-month RCT with a 24-month follow-up. Participants will be randomly allocated into either a health and PA counselling group (intervention) or relaxation group (control intervention). All participants will receive five group specific face-to-face counselling sessions and 11 phone calls. The counselling intervention will include individualised health counselling, strength and balance training, and guidance to regular PA. The control group will receive relaxation exercises. Outcomes will be assessed at baseline, 12, 24 and 48 months. Primary outcomes are average life-space mobility score and falls rates. Life-space mobility will be assessed by a validated questionnaire. Falls rates will be recorded from fall diaries. Secondary outcomes are data on fall-induced injuries and living arrangements, number of fallers, fracture risk, mean level of PA, physical performance, quality of life, mood, cognition, balance confidence and fear of falling. Data will be analysed using the intention-to-treat principle. Costeffectiveness of the programme will be analysed. Ancillary analyses are planned in participants with greater adherence. Ethics and dissemination Ethical approval was obtained from the Ethics Committee of the Tampere University Hospital (R15160). Outcomes will be disseminated through publication in peer-reviewed journals and presentations at international conferences.

Trial registration ISRCTN65406039; Pre-results.

\section{INTRODUCTION}

The disablement process model by Verbrugge and Jette ${ }^{1}$ describes the path from pathology

\section{Strengths and limitations of the study}

- This randomised controlled trial will investigate the effectiveness of a pragmatic home-based exercise programme on life-space mobility and falls rates.

- The counselling protocol is delivered by nurses and physiotherapists according to current evidence-based principles to maximise long-term exercise adherence and commitment to physical activity, and to prevent falls.

- Counselling sessions take place at easily accessible community-based Health Kiosk and/or Service Centre environment.

- This will be the first randomised controlled trial to evaluate the effectiveness of health and physical activity counselling in a community-based environment to improve life-space mobility and prevent falls.

- Research nurses and research physiotherapists are not blinded to the random allocation.

to disability via impairments and functional limitations. Accordingly, multiple health conditions (eg, osteoarthritis) may lead to physical impairments (eg, weak leg extensor muscles), which may result in functional limitations (eg, challenges with lower extremity function and balance). Functional limitations may finally lead to disability resulting in uncertainty to walk safely, an increased fear of falling and rate of falls, all of which can further reduce movement within a typical living area. ${ }^{23}$ In addition, restricted life-space mobility can reduce participation in social activities, which can lead to little utilisation of community amenities available. This vicious cycle can escalate as overall health and well-being of older adults deteriorates.

Developing and implementing effective strategies that prevent disability and falls among older people is an urgent public 


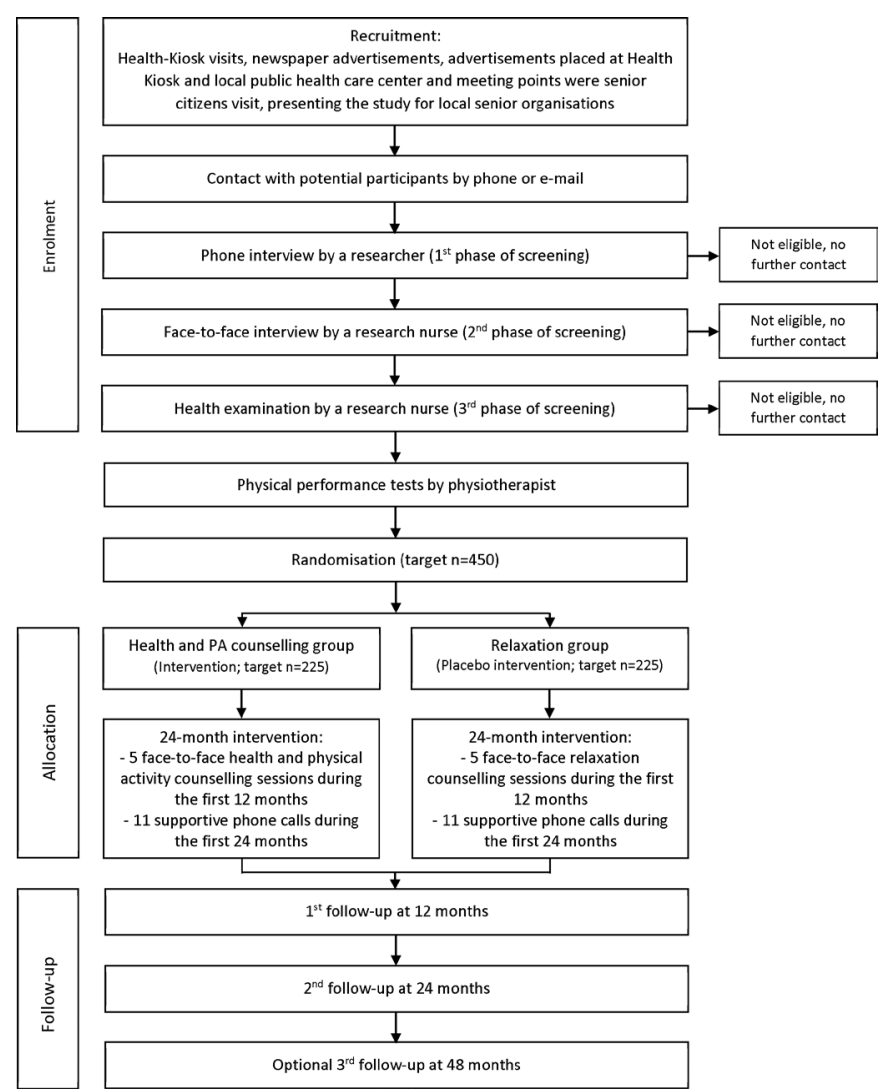

Figure 1 Flow chart of the COSMOS study. PA, physical activity.

health issue given our ageing population and the personal and societal impact from falls. Targeted exercise programmes including muscle strength and balance training, such as the Otago Exercise Program, have been found to be effective at preventing falls and injurious falls among community-dwelling older adults. ${ }^{4-6}$ There is also evidence that older people with multiple risk factors for falls and thus at high risk of falling benefit from a multifactorial approach. ${ }^{4}$ For instance, a previous multifactorial trial (Chaos Falls Clinic), which included an individualised 12 month falls prevention programme, in high-risk individuals aged 70 years or over reduced falls and fall-induced injuries by over $25 \% .{ }^{7}$ Despite its effectiveness, multifactorial interventions can be expensive and labour-intensive.

Community-based and easily accessible service platforms and concepts provide an opportunity potential for health and physical activity counselling since they may reach a broad range of older people who already wish to change their lifestyle. As an approach to reform the social and healthcare system in Finland and confront European megatrends such as the ageing population with increasing public costs, community-based Health Kiosks and Service Centres have been launched to enable rapid health screening and counselling to support people to be active and participative in the society. Their focus is on health promotion and disability prevention. Scheduled appointments are not required and they are free of charge. A rapid health screening with tailored counselling and guidance at an easily accessible environment can offer a modernised primary care concept to tackle or slow down progressive but early phase health issues and disablement processes. It may also provide a unique opportunity to increase physical activity, support physical function, and avoid falls, depressive symptoms and social isolation. ${ }^{89}$

To our knowledge, only one previous randomised controlled trial has shown the impact of a multifactorial intervention on life-space mobility in older people. ${ }^{10}$ It has been recommended that future studies should measure mobility at both the participation and activity levels. In addition, it has been suggested that future research should include a longer follow-up period to determine if the benefits of any interventions are maintained long term (>12 months). Therefore, COSMOS will be the first randomised controlled trial to evaluate the effectiveness of a 24 month health and physical activity counselling programme in a community-based Health Kiosk and/or Service Centre environment to improve life-space mobility and physical activity and prevent falls, and evaluate whether any benefits are sustained after a 24-month follow-up. Another novel aspect is that this study will assess simultaneously changes in the ratio of falls rates and the difference in rate changes in the lifespace mobility outcome.

\section{Trial objectives and hypothesis}

The primary aim of this randomised controlled trial (RCT) named 'Counselling for physical activity, life-space mobility and falls prevention in old age' (COSMOS) is to examine the effectiveness of a 24-month community-based health and physical activity counselling programme in increasing life-space mobility and reducing the rate of falls in community-dwelling elderly people.

Secondary aims of the study are to evaluate the effects of the counselling intervention on data on fall-induced injuries and living arrangements, number of fallers, fracture risk, mean level of physical activity, physical performance, quality of life, mood, cognition and balance confidence. We will also evaluate the cost-effectiveness of the counselling programme within the community-based environment for falls and whether any of the aforementioned potential benefits can be maintained 2 years after the end of the 24-month intervention.

We hypothesise that (1) life-space mobility can increase and (2) fall rates can reduce via improved lower extremity ability, balance and mobility. These together enable increasing walking distances and thus support safe attendance to physical and social activities outside one's own neighbourhood or home district.

\section{METHODS AND DESIGN}

This protocol article is written based on the SPIRIT reporting guidelines. ${ }^{11}$ The experimental design is illustrated in figure 1. 


\section{Trial design and study setting}

COSMOS is a pragmatic single-blinded 24-month RCT with a 24-month follow-up at a community-based environment. Participants will be randomised into one of two groups: (1) a health and physical activity counselling intervention or (2) a relaxation intervention (control). All participants will be assessed at baseline and after 12 and 24 months. In addition, there will be follow-up assessments at 48 months. All assessments will begin with a structured interview and health examination performed by a research nurse and followed by physical performance tests carried out by a research physiotherapist. All assessments and intervention sessions will take place at the Health Kiosk of Ylöjärvi or at the Service Centre of Ylöjärvi, Finland. Health Kiosk is a nurse-led pilot primary care service environment situated in a shopping centre. ${ }^{89}$ Service Centre is a modern meeting place for senior citizens with various indoor and outdoor activities. Ylöjärvi is a municipality of 32000 inhabitants including suburban and rural areas. Study participants can choose themselves the place they would prefer to visit.

\section{Participant eligibility}

The target number of participants is 450 who will be randomly allocated to each group ( $\mathrm{n}=225 \mathrm{each})$. Both men and women will be recruited. Participant inclusion criteria are as follows: (1) aged 65 years or over; (2) community-living people; (3) living in Pirkanmaa District, Finland; (4) at least minor self-reported mobility difficulty.

Mobility difficulty will be assessed by using a structured and validated interview asking each participant about his or her ability to walk $2.0 \mathrm{~km}$, walk $0.5 \mathrm{~km}$ and climb up one flight of stairs. ${ }^{12}$ The questions are formulated as follows: "Do you have difficulty in ..." with five alternative response options provided: (1) ... able to manage without difficulty; (2) ... able to manage with some difficulty; (3) ... able to manage with great deal of difficulty; (4) ... able to manage only with help of another person; (5) ... unable to manage even with help. To identify persons with minor mobility difficulty, additional questions are posed to participants who do not report task difficulty with any of the above questions. The questions concern the modification of task performance and the alternatives given are resting in the middle of the performance, using an aid, taking support from handrails, having reduced the frequency of performing the task, having slowed down performance of the task, experiencing tiredness when performing the task or some other change in carrying out the task. Minor mobility difficulty is considered if participant reports task modification in one or more of the tasks listed above.

Participant exclusion criteria are (1) severe functional limitations (unable to walk $500 \mathrm{~m}$ unaided), (2) severe cardiovascular or pulmonary disease, (3) severe progressive disease, (4) terminally ill (predicted life expectancy $<12$ months), (5) memory impairment (MMSE score 21 points or less), ${ }^{13}$ (6) living in an institution, (7) unwilling to be randomised or $(8)$ alcoholism (AUDIT score $\geq 15$ ). ${ }^{14}$
Severe cardiovascular and severe pulmonary disease is defined as conditions which are currently either unstable or contraindications for physical exercise and/or need immediate medical attention. Severe progressive disease is defined as conditions such as neoplasm and amyotrophic lateral sclerosis, which have poor prognosis and presumably poor response or no response to physical exercise.

\section{Recruitment}

We will recruit eligible men and women during their Health Kiosk and Service Centre visits as well as via newspaper advertisements, notice boards, community centres and at senior events. All participants will be initially screened for eligibility over the telephone (age, living arrangements and place of residence) where they will have the opportunity to ask questions and have an informed discussion with research staff. Following the telephone screening, those who are eligible and are willing to participate will receive an information letter, consent forms and reply-paid envelope. On receiving a signed informed consent form, a member of the research team will sign each form prior to the baseline assessments. Potential participants will be invited to the baseline assessments, where a trained research nurse confirms their eligibility with a structured interview and health examination.

\section{Random allocation}

Participants will be randomly allocated into either (1) the health and physical activity counselling intervention or (2) the relaxation intervention (control group). A computer-generated randomisation protocol will be created by a statistician who is not part of the research team. Random allocation will be stratified by sex, age (65-79 years/80 years or older) and presence or absence of falls during the last 24 months. Block size of 6, 8, 10 or 12 will be randomly varied to ensure the equality of group sizes (allocation ratio 1:1). Allocation results will be stored in sealed envelopes and stored in locked cabin. After the baseline measurements, a researcher will open one envelope according to each participant's sex, age and previous falls, and then verify with the research records which intervention the participant is allocated. Participants are informed whether they belong to the health and physical activity counselling or relaxation group. Allocation concealment will be ensured, as the randomisation code will only be released at the completion of the study. Research nurses and physiotherapists are not blinded to the group allocation due to limited financial and personnel resources. The principal investigator will be blinded.

\section{Interventions}

The COSMOS study involves two interventions: (1) health and physical activity counselling, and (2) a relaxation intervention (control group). Online supplementary figure describes the participant timeline. Both interventions include five face-to-face sessions taking place 
at week 1 and 1, 3, 6 and 12 months after the baseline measurements. During each face-to-face session, a physiotherapist will provide instructions for the next level of the programme. Participants will be provided with 11 supportive telephone calls by a physiotherapist, regardless of the intervention, which will be delivered at 2, 4, 5 , $7,8,9,10,11,16,20$ and 23 months after baseline. The total duration of the interventions is 24 months. At the end of the first face-to-face session, the physiotherapist informs the participant on how to fill out the physical activity and falls diary.

\section{Health and physical activity counselling intervention}

Participants randomised to the health and physical activity counselling intervention will receive five individually tailored 1.5-hour face-to-face sessions containing a $30 \mathrm{~min}$ health counselling session by a trained research nurse together with a 60 min physical activity counselling session delivered by an experienced research physiotherapist.

The health counselling follows the motivational interviewing concept ${ }^{15}$ based on the Social Cognitive Theory, ${ }^{16}$ and the trans-theoretical model. ${ }^{17}$ The structure of the health counselling is based on the guidelines of the IKINÄ manual, which is a guide for preventing falls and harm from falls in older people, released by the Finnish National Institute for Health and Welfare ${ }^{18}$ Accordingly, during health counselling sessions, the nurse will advise participants on safety issues related to their home environment, such as providing recommendations to use anti-slippery shoe devices during winter, and participating in regular physical activity. In addition, participants in the health and physical activity counselling intervention will receive handouts on how to avoid fall accidents in the home environment and outdoors. Moreover, health counselling sessions will include counselling on a healthful diet and recommendations to reduce alcohol consumption and smoking based on discussions with each participant about her/his background and habits, and motivation to change. ${ }^{15}$ The nurse will also discuss topical and relevant health-related issues with health and physical activity counselling intervention members, that is, managing blood pressure, medication and depressive mood.
The physical activity counselling is based on the modified version of the Otago Exercise Program (OEP, available online) ${ }^{19}$ The OEP is an innovative model of low-frequency physical activity counselling and exercise training tailored for older people and typically delivered by a physiotherapist at older people's home. It contains four levels (A, B, C and D) which all contain strengthening exercises for lower extremity muscles as well as balance, walking and stair climbing exercises and active range of movement exercises (eg, neck rotations and hip and knee extensions). The exercises on each level take about 30 min to complete. Participants are expected to exercise three times a week at home and go for a walk at least twice a week for $30 \mathrm{~min}$. Walking exercise can also be broken into smaller periods for example, three 10 min bouts.

The physiotherapist may modify and apply the OEP individually based on health, motivational status and participant goals. The participants will receive progressive illustrated instructions and will be provided with ankle weights $(0.5-5.0 \mathrm{~kg})$ for the first 12 months. Thereafter, the therapist will encourage participants to attend a local gym or be involved with other community exercise facilities. For this study, two additional training levels (COSMOS 1 and COSMOS 2, illustrated in table 1) have been developed to ensure progression throughout the 24-month intervention.

During the physical activity counselling sessions, a physiotherapist will also discuss the importance of regular and diverse physical activity and presents the Physical Activity Pie for Older Adults (Finnish recommendations for physical activity among 65 years old and older) (http:// www.ukkinstituutti.fi/filebank/64-physical_activity_pie. pdf). In addition, the therapist will provide an exercise referral to a local community exercise facilities based on the earlier discussions with the participant about her/his background and motivation to exercise. When the participant receives a referral to a community-based exercise programme, the physiotherapist will instruct him/her to replace one of the weekly Otago, COSMOS or walking exercises with corresponding exercise. For instance, the participant may replace the Otago strength exercise with gym training or by attending a strength-training group.

Table 1 Content of the COSMOS 1 and 2 levels

Cosmos $1 \quad$ cosmos 2

\begin{tabular}{|c|c|c|}
\hline Warm-up & Same as in the Otago Exercise Program & Same as in the Otago Exercise Program \\
\hline Strengthening exercises & $\begin{array}{l}\text { One-legged squat } \\
\text { One-legged sit to stand } \\
\text { Sideways squats } \\
\text { Jumping exercises }\end{array}$ & $\begin{array}{l}\text { Same strengthening exercises as in the COSMOS } \\
\rightarrow \text { Repetitions and series are updated and jumping } \\
\text { exercises are extended and more demanding }\end{array}$ \\
\hline Balance exercises & $\begin{array}{l}\text { Same as in the D level of the Otago } \\
\text { Exercise Program but stair climbing is } \\
\text { replaced with squats } \\
\text { In addition, multitasking is incorporated } \\
\text { into all exercises (eg, participants count } \\
\text { repetitions or seconds backwards) }\end{array}$ & $\begin{array}{l}\text { Same balance exercises as in the COSMOS } 1 \text { level, } \\
\text { but all exercises are performed with eyes closed }\end{array}$ \\
\hline
\end{tabular}


Correspondingly, the participant may replace Otago balance exercise with yoga, Pilates, Tai Chi or other guided balance exercise. Walking exercises can also be replaced, for example, with swimming or other aerobic exercise format.

\section{Relaxation intervention (control group)}

Participants randomised to the relaxation intervention will receive five $45 \mathrm{~min}$ face-to-face sessions of structured relaxation exercises instructed by a physiotherapist. We believe that offering relaxation exercises will motivate the control participants to continue in the study without increasing their physical activity. The relaxation programme will be updated during each face-to-face session and will proceed as follows: (1) learning the diaphragmatic respiration technique, (2) learning the tension-relaxation technique, (3) using tension-relaxation technique, (4) using techniques learnt in previous exercises to whole-body relaxation and (5) learning consciousness of the body sensations. All exercises will be displayed on a compact disc or via mp3 format. In addition, written instructions will be available. During the first face-to-face session, participants will receive the same handouts as the health and physical activity counselling intervention members on how to avoid fall accidents in the home environment and outdoors.

\section{Supportive telephone calls}

During 11 supportive telephone calls, the physiotherapist will enquire about how exercise (physical activity or relaxing programme) is progressing, has the participant fallen and ensure that the most recent fall and exercise diary is returned. In addition, therapists will confirm or schedule the next face-to-face session or 12-month and 24-month follow-up measurements when appropriate. If a participant has fallen, therapists will confirm the circumstances and consequence related to the fall/falls. For those in the health and physical activity counselling group, the therapist will also discuss if there is a need to update the programme, that is, revise the number of repetitions and/or series or change the magnitude of the ankle weight before the next face-to-face session. In addition, any barriers to exercise that have come up from the participants will be addressed.

\section{Outcomes}

Assessments will include a comprehensive battery of tests and questionnaires on mobility, physical activity, physical function and health. The baseline assessment will take about 2 hours to complete whereas 12-month and 24-month assessments will take about 1.5 hours. The order of the assessments and measurements is standardised at each time point. Table 2 presents the outcome and other variables, methods and schedule of the assessments in the study.

\section{Primary outcomes}

Daily filled and monthly returned fall diaries will be used to gather information on the falls rates during the 24-month intervention and follow-up. A fall is defined as an unexpected event in which participant comes to rest on the ground, floor or other lower level. ${ }^{20} \mathrm{~A}$ research physiotherapist will phone monthly all those participants who have reported a fall or falls or if a diary is not returned.

Life-space mobility assessment (LSA) is determined from a validated questionnaire, which measures the size of the area that a person has moved around in during the 4 weeks preceding the assessment. ${ }^{2}$ It correlates with observed physical performance and self-reported function. ${ }^{2}$ For each level of life-space (bedroom, other rooms, outside home, neighbourhood, town, beyond town), persons are asked how many days within a week they attained that level of life-space and whether they need help from another person or from assistive devices. A composite measure of life-space combines the components of life-space level attained, degree of independence and frequency of attainment. ${ }^{3}$

\section{Secondary outcomes}

A number of secondary outcome measures will be assessed to clarify potential mechanisms underlying any reduction in fall rates or increased life-space mobility during the trial, and to determine to what extent the training transfers to other important outcomes.

Physical activity: The Finnish Hookie AM 20 triaxial accelerometer will be used to measure all physical activity over a 7-day period. The Hookie AM 20 device and related data analyses are based on the UKK Institute's algorithms which has been used in three large Finnish population-based cohort studies,${ }^{21}{ }^{22}$ and in older community-dwelling individuals. ${ }^{23}$ A physical activity and exercise diary will also be used during the first 24 months' period of the study. Self-reported physical activity will also be quantified using a modified version of the scale by Grimby. ${ }^{24}$

Physical performance: An experienced research physiotherapist will conduct all physical performance tests, including the Timed Up and Go test ${ }^{26}$ and Short Physical Performance Battery. ${ }^{27}$ Handgrip strength from the dominant arm will be assessed using the Jamar hand dynamometer. ${ }^{28}$

A fall indicator variable (yes/no) will be formed. In addition, fall-induced injuries will be assessed based on diaries filled daily and returned each month until 24 months after the baseline. Hospital registers will also be used to verify severe injuries (ie, fractures and head injuries) during the intervention and follow-up. Injuries will be categorised as follows: (1) soft-tissue bruises and contusions, (2) wounds and lacerations, (3) bone fractures, (4) joint distortions and dislocations, (5) head injuries other than fractures and (6) other injuries. In addition, all injuries will be categorised based on medical contact and/or treatment.

Health-related quality of life will be assessed using the WHO Quality of Life (WHOQOL) 26-item short version questionnaire, which includes questions related to 
Table 2 Outcome and other variables, methods and schedule of the assessments

\section{Continuous monitoring}

Falls rates

Daily filled and monthly returned diaries

Number of fallers i.e. a fall indicator variable (yes/no)

Daily filled and monthly returned diaries

Fall-induced injuries

Daily filled and monthly returned diaries and telephone interviews

Hospital registers are used to verify severe injuries

Health service use

Hospital registers are used to verify severe injuries

Adverse events due to interventions

Daily filled and monthly returned diaries and telephone interviews

Participant adherence to the interventions

Average number and duration of exercise sessions and total $\mathrm{N}$ number and duration of exercise sessions based on daily filled and monthly

Perceived exertion of interventions

Modified Borg scale (range 0-10)

Physical, cognitive and social assessments

Physical activity Hookie AM 20 triaxial accelerometer for 7 days

Daily filled and monthly returned physical activity and exercise $\mathrm{N}$ diaries

Validated questionnaire (Scale of Grimby)

BL

12-month

24-month

48-month

$\mathbf{O}$

$\mathbf{P}$

$\begin{array}{llll}N & Y & Y & Y\end{array}$

$\begin{array}{llll}N & Y & Y & Y\end{array}$

S

$\begin{array}{llll}N & Y & & \end{array}$

N

Y

Y

Y

N

Y

Y

Y

N

Y

Y

N

N

Physical performance $Y$

N

$Y$

$\mathrm{Y}$

$\mathrm{N}$

$\mathrm{N}$

BL

12-month

24-month

48-month

0

$\mathrm{S}$

$\begin{array}{llll}Y & Y & Y & Y\end{array}$

$\mathrm{Y}$

$\begin{array}{llll} & Y & Y & \\ & Y & Y & Y\end{array}$

Timed Up and Go-test (TUG)

Short Physical Performance Battery (SPPB)

Jamar hand dynamometer

$\begin{array}{ll}Y & Y \\ Y & Y \\ Y & Y\end{array}$

$\mathrm{Y}$
$\mathrm{Y}$
$\mathrm{Y}$

Body composition

Height and weight are measured and BMI is calculated

Y

Y

Y

S

Fracture risk

World Health Organization Fracture Risk Assessment Tool (FRAX)

Y

Y

Y $\quad Y$

Cardiovascular condition

New York Heart Association functional class (NYHA)

Orthostatic test

$\begin{array}{llll}Y & Y & Y & Y \\ Y & Y & Y & N\end{array}$

Self-reported physical ability

Determined by asking

Y

Y

Y

Y

Mobility difficulty

\section{Structured interview}

Y

Y

Y

Y

Need of mobility assistive devices

Determined by asking

Living arrangements

Y

Y $\quad$ Y

Y $\quad$ Y

Y

Determined by asking

Y

Y

Y

Y 


\begin{tabular}{|c|c|c|c|c|c|}
\hline Questionnaire-based assessments & BL & 12-month & 24-month & 48-month & $\mathbf{O}$ \\
\hline Life space mobility & & & & & $\mathbf{P}$ \\
\hline Life-space mobility assessment (LSA) & Y & Y & Y & Y & \\
\hline Balance confidence & & & & & $\mathrm{S}$ \\
\hline Activities-specific Balance Confidence scale (ABC) & Y & Y & Y & Y & \\
\hline Fear of falling & & & & & $\mathrm{S}$ \\
\hline Determined by asking and by Visual Analogue Scale (VAS) & Y & Y & $\mathrm{Y}$ & $\mathrm{Y}$ & \\
\hline Quality of life (QOL) & & & & & S \\
\hline $\begin{array}{l}\text { World Health Organization Quality of Life (WHOQOL) } \\
\text { questionnaire }\end{array}$ & $\mathrm{Y}$ & Y & $\mathrm{Y}$ & Y & \\
\hline Cognitive status & & & & & $\mathrm{S}$ \\
\hline Mini-Mental State Examination (MMSE) & Y & Y & Y & $\mathrm{Y}$ & \\
\hline Depressive mood & & & & & $\mathrm{S}$ \\
\hline Geriatric Depression Scale (GDS-15) & Y & Y & Y & Y & \\
\hline \multicolumn{6}{|l|}{ Alcohol consumption } \\
\hline The Alcohol Use Disorders Identification Test (AUDIT) & $\mathrm{Y}$ & $\mathrm{Y}$ & $\mathrm{Y}$ & Y & \\
\hline
\end{tabular}

$\mathrm{BL}$, baseline; N, no; O, outcome;P, primary; S, secondary; Y, yes.

physical health, psychological health, social relationships and environment. ${ }^{29}$ Living arrangements will be determined by interview. Fracture risk will be assessed by WHO Fracture Risk Assessment Tool (FRAX) via interview. The FRAX algorithms give the 10-year probability of hip fracture and a major osteoporotic fracture (clinical spine, forearm, hip or shoulder fracture).$^{30}$

Depressive mood will be assessed using the Geriatric Depression Scale (GDS-15) ${ }^{31}$ Participants who achieve 6 points or more on GDS-15 test will be referred to their physician for follow-up. Cognitive status will be assessed via the Mini-Mental State Examination (MMSE) ${ }^{13}$ Participants who score 21 points or less in MMSE are excluded and referred to a physician appointment.

Balance confidence will be evaluated using the Activities-specific Balance Confidence scale. ${ }^{32}$ Fear of falling will be assessed (yes/no) and measured by the Visual Analogue Scale. ${ }^{33}$ A $100 \mathrm{~mm}$ long line will be used with the left end of the line $(0 \mathrm{~mm})$ representing 'no fear' and the right end $(100 \mathrm{~mm})$ 'extreme fear'.

\section{Other variables}

During the health examination, the research nurse will measure height and weight using standard procedures. Body mass index will be calculated as body weight $(\mathrm{kg}) /$ height $(\mathrm{m})$ squared. The research nurse will also ask about any chronic and geriatric conditions, prescription medication(s) and the presence of any cardiovascular condition using New York Heart Association functional class, ${ }^{34}$ and perform an orthostatic test. ${ }^{35}$ Alcohol consumption will be assessed by the AUDIT-C tool and additionally by AUDIT if the AUDIT-C score is 6 or more among men and 5 or more among women. ${ }^{14}$ If the AUDIT score is 15 or more, participants will be excluded and referred to a healthcare practitioner.

Self-reported physical ability will be determined via interview and asking participants: "How would you describe your physical ability?" Options are (1) excellent, (2) good, (3) average and (4) poor. Need of mobility assistive devices will also be determined via interview. Mobility difficulty will be assessed using a structured interview described earlier (see participant eligibility). As an outcome measure of adherence, we use the average number and duration of exercise sessions and total number and duration of exercise sessions based on daily completed and monthly returned physical activity and exercise diaries. In addition, perceived exertion will be assessed using the modified Borg Rating of Perceived Exertion scale (range, 0-10). ${ }^{36}$

Demographics which include age, sex, marital status, education and most recent occupation, as well as diet, use of spectacles and smoking habits, and whether participants have any problems related to vision and hearing, will be determined by interview. Previous falls will also be asked at baseline: "Have you fallen (and if so, how many times) during the previous year $/ 6$ months/month (without substantial external force) and did you injure yourself? Adverse events due to interventions are assessed by daily completed and monthly returned diaries and telephone interviews.

\section{Statistical methods}

Pretrial power calculations

We estimated the minimum required sample size in a simulation model including the continuous and count outcomes and the mutual correlation estimated via normally distributed random effects. Sample size estimation accounted 
for the multiple testing and the correlation between outcomes. ${ }^{37}$ Based on previous research, ${ }^{7}$ we assumed that the control group would have a fall incidence of 131 per 100 person-years and the corresponding rate in the intervention group would be 118 per 100 person-years, which corresponds to a modest relative risk reduction of about $10 \%$ in favour of the intervention group. To allow some overdispersion in the fall count, the normally distributed random effect variance was set at 0.3. Based on data from the Life-Space Mobility in Old Age (LISPE) study, ${ }^{38}$ we set the mean at 64.0 (SD 20.6) for the life-space mobility score, which was increased to 70.4 in the intervention group during the follow-up representing a relative increase of $10 \%$. To obtain a conservative sample size estimate, the random effect correlation was set at the low value 0.10 . The simulation studies were based on 1000 replications of the model parameters. To find the likelihood ratio test statistic significant at 5\% significance level for the above mean difference and risk ratio simultaneously, power of $80 \%$ was reached with a sample size of 346 based on equal allocation of subjects into the control and intervention groups. To account for $30 \%$ attrition, the sample size was increased to 450 (225 in each group). In previous intervention studies including similar components, drop-out rates have been approximately $15 \%$. $^{7}$ We hypothesised the attrition rate to be even greater because for majority of the participants, participation involved travelling across Pirkanmaa District (distances were even $100 \mathrm{~km}$ in each direction), and travelling costs were not covered and transportation was not arranged by COSMOS.

\section{Statistical analyses}

All statistical analyses will be conducted using the Mplus software and IBM SPSS software package V.24. We will analyse the data on an intention-to-treat basis, using the data from all randomised participants despite the protocol adherence and independent of the sponsor and competing interest. Follow-up time for falls, fallers and fall-induced injuries including fractures will be calculated from the day when the participant started the intervention to the end of the 24-month intervention +24-month follow-up or withdrawal from study.

The primary outcome analysis is a likelihood-ratio test assessing simultaneously changes in the ratio of falls rates and the difference in rate changes in the LSA outcome. The test is based on a model of the fall outcome in a negative binomial regression model, where a random effect is used to account for likely overdispersion in the fall count distribution and the intraclass correlation of the measurement time points. Descriptive information is calculated as incidence rates of falls, fallers, multiple fallers and fall-induced injuries (including fractures) per 100 person-years. Proportion of fallers between groups will be reported using incidence rate ratio statistics.

Ancillary analysis using causal modelling will be conducted to establish intervention effects in people with greater adherence (per protocol analysis). Covariance analysis will be used to analyse between-group differences in other continuous variables and general linear models will be used to assess the effect of group allocation on continuous secondary outcome measures. Logistic regression models will be used to compare the two intervention groups on dichotomous outcome measures. The explanatory factors of exercise adherence will be investigated in a longitudinal path model enabling the linking data from individual characteristics to intervention effectiveness. Directed acyclic graphs are used to establish theoretical model relationships that serve as basis for model development with observed data. In addition, we model physical activity trajectories and investigate individual variability among the trajectories.

Economic analyses will be approached from the perspective of the community healthcare provider. The health outcome measure will be cost per fall prevented over the study duration. Costs will include intervention costs as well as fall-induced healthcare and community service costs. Cost utility is based on quality-adjusted life years (QALY) gained, where quality is measured at 12, 24 and 48 months with the WHOQOL index. Using mean costs and QoL in each treatment arm, the intervention cost-effectiveness will be assessed by comparing the intervention incremental cost per a prevented fall and incremental cost per QALY gained to those in the control group. The probability that the intervention is cost-effective will be computed based on bootstrapping the incremental cost-effectiveness ratio as described in Gray et al..$^{39}$ Cost-effectiveness acceptability curves will be plotted for various levels of willingness to pay.

Where missing data are generated through the missing-at-random (MAR) mechanism, we will employ the standard MAR-based likelihood specification in Mplus. ${ }^{40}$ A custom missing data model will be used when missing data are generated by a non-random mechanism.

\section{Data management}

Once a participant has been randomly allocated, every effort will be made to follow up the participant on outcome measures until the end of the study period. Any participants who discontinue or deviate from the intervention protocols or fail to complete the exercise and falls diary will still be invited to complete the 12-month and/or 24-month follow-up measurements. Participant data are stored on a secure database in accordance with the General Data Protection Regulations (2018). All collected data will be coded with unique identification numbers and stored centrally on the secure database of the University of Jyväskylä, a password-protected computer or in a locked filing cabinet in a secure office space, only accessible by a limited number of people. The questionnaires and forms will be checked for completeness and congruity instantly when filled and/or received and again before data entry onto the database. In addition, we will regularly check the data files for omissions and errors to ensure the data integrity. Trial documentation and data will be archived for at least 10 years after completion of the trial after which it will be destroyed. 
The data monitoring committee consists of the research group members (see front page).

\section{Trial monitoring}

A standard operation procedure has been written before launching the study and will be followed carefully throughout the study. Regular meetings will be organised for monitoring the quality of data collection. Senior researchers will carefully educate the personnel performing the measurements and the same staff will engage in the data collection throughout the study.

\section{Ethics and dissemination}

The Ethics Committee of the Tampere University Hospital has approved the procedures and design of the trial (3 November 2015, ref: R15160). The COSMOS study is carried out according to the guidelines of good scientific practice (Declaration of Helsinki). Any protocol modifications will be reported to the Ethical Committee and to the trial registry (ISRCTN). Participants are insured for intervention-related harms. Moreover, we will record any adverse events from either of the interventions and report serious adverse events to the ethics committee. Participants may withdraw from the study for any reason at any time.

The research team is committed to full disclosure of the results of the trial. Findings will be reported in accordance with the CONSORT guidelines in peer-reviewed journals and international scientific conferences. The funder will have no role in the analysis or interpretation of the trial results. The study results will also be disseminated to the participants. Two information sessions will be organised to the study participants when the data of the primary outcomes have been analysed.

The research environment of the COSMOS trial is unique because the trial is conducted at a Health Kiosk and/or a Service Centre, which are new, easily accessible, free-of-charge counselling concepts, targeted and tailored for elderly people. This allows extending the study further to investigate the effectiveness of the counselling and exercise referral to promote actual mobility and to prevent fractures as a primary endpoint, which, according to our knowledge, has not been done before. If proven safe and effective in the population setting, the counselling/referral concept could also be modified and extended to investigate other health hazards such as elderly people experiencing memory complaints or cognitive impairments and/or people having early depressive signs to meet their hazards early for effective prevention and/or treatment.

\section{Patient and public involvement statement}

We did not directly include patient and public involvement in this study, but we will develop the counselling programme based on participant feedback.

\footnotetext{
Author affiliations

${ }^{1}$ Faculty of Sport and Health Sciences, University of Jyväskylä, Jyväskylä, Finland ${ }^{2}$ Faculty of Sport and Health Sciences, Gerontology Research Center, University of Jyväskylä, Jyväskylä, Finland

${ }^{3}$ UKK Institute for Health Promotion Research, Tampere, Finland
}

${ }^{4}$ Institute for Physical Activity and Nutrition, Deakin University, Geelong, Victoria, Australia

${ }^{5}$ Department of Orthopaedics and Traumatology, Tampere University Hospital, Tampere, Finland

${ }^{6}$ Research and Development, School of Health and Social Studies, Jyväskylä University of Applied Sciences, Jyväskylä, Finland

${ }^{7}$ Central Finland Centre for Economic Development, Transport and the Environment, Jyväskylä, Finland

${ }^{8}$ GeroCenter Foundation for Aging Research and Development, Jyväskylä, Finland

Acknowledgements We would like to thank all the trial participants. We also thank research physiotherapists Elina Ahlstedt-Kivelä, Paju Becker and Eerika Tiirikainen as well as research nurses Anneli Peltonen, Kirsi Perkkiö, Tiina Raittinen, Laura-Emilia Vainio and Arja Ylä-Rakkola for their valuable contribution; physician Jaakko Valvanne for his feedback on the health examination concept and psychologist Päivi Lappalainen for introducing the motivational interviewing technique to the study personnel; statistician Markku Kauppinen, who created the random allocation protocol; Sirpa Ellala, Paula Pasanen-Aro, Maarit Pitkänen, Anne Santalahti and Laura Tyyskänen at the administration of the City of Ylöjärvi for ongoing collaboration; and the Academy of Finland for funding the study.

Contributors Authorship is based on the ICMJE Recommendations (2013). RN is the responsible investigator who conceived the study and will oversee the data collection. The study was conceived with input from TaR, RD, UMK, TT, HS, PK, AH, SS, LK, TiR and OT. JE and SK are responsible for managing the data collection. JE wrote this protocol manuscript, the final version of which all other authors have revised and provided input according to their area of expertise. All authors approved the final version of the manuscript.

Funding This work was supported by the Academy of Finland (grant no. 289523).

Competing interests RN, University of Jyväskylä and GeroCenter Foundation, Jyväskylä, Finland, is a co-owner of the Devisys 0y, Tampere, Finland, a company that produces anti-slip devices for winter conditions.

Patient consent for publication Not required.

Provenance and peer review Not commissioned; externally peer reviewed.

Open access This is an open access article distributed in accordance with the Creative Commons Attribution Non Commercial (CC BY-NC 4.0) license, which permits others to distribute, remix, adapt, build upon this work non-commercially, and license their derivative works on different terms, provided the original work is properly cited, appropriate credit is given, any changes made indicated, and the use is non-commercial. See: http://creativecommons.org/licenses/by-nc/4.0/.

\section{REFERENCES}

1. Verbrugge LM, Jette AM. The disablement process. Soc Sci Med 1994;38:1-14.

2. Baker PS, Bodner EV, Allman RM. Measuring life-space mobility in community-dwelling older adults. J Am Geriatr Soc 2003;51:1610-4.

3. Peel C, Sawyer Baker P, Roth DL, et al. Assessing mobility in older adults: the UAB study of aging life-space assessment. Phys Ther 2005;85:1008-119.

4. Gillespie LD, Robertson MC, Gillespie WJ, et al. Interventions for preventing falls in older people living in the community. Cochrane Database Syst Rev 2012:CD007146.

5. El-Khoury F, Cassou B, Charles M-A, et al. The effect of fall prevention exercise programmes on fall induced injuries in community dwelling older adults: systematic review and metaanalysis of randomised controlled trials. BMJ 2013;347:f6234.

6. Guirguis-Blake JM, Michael YL, Perdue LA, et al. Interventions to prevent falls in older adults: updated evidence report and systematic review for the US Preventive Services Task Force. JAMA 2018;319:1705-16.

7. Palvanen M, Kannus P, Piirtola M, et al. Effectiveness of the chaos falls clinic in preventing falls and injuries of home-dwelling older adults: a randomised controlled trial. Injury 2014;45:265-71.

8. Kork A, Kivimäki R, Rimpelä A, et al. Terveyskioski palveluinnovaationa - Lahden terveyskioski-hankkeen väliarviointi (Vaihe II) (in Finnish). Sitra 2011;35.

9. Kork A, Vakkuri J. Matalan kynnyksen palvelumalli perusturvan palvelualueella (in Finnish). Sitra 2013;23.

10. Fairhall N, Sherrington C, Kurrle SE, et al. Effect of a multifactorial interdisciplinary intervention on mobility-related disability in frail older people: randomised controlled trial. BMC Med 2012;10. 
11. Chan A-W, Tetzlaff JM, Altman DG, et al. Spirit 2013 statement: defining standard protocol items for clinical trials. Ann Intern Med 2013;158:200-7.

12. Mänty $M$, Heinonen $A$, Leinonen $R$, et al. Construct and predictive validity of a self-reported measure of preclinical mobility limitation. Arch Phys Med Rehabil 2007;88:1108-13.

13. Folstein MF, Folstein SE, McHugh PR, et al. A practical method for grading the cognitive state of patients for the clinician. J Psychiatr Res 1975;12:189-98.

14. Saunders JB, Aasland OG, Babor TF, et al. Development of the Alcohol Use Disorders Identification Test (AUDIT): WHO Collaborative Project on Early Detection of Persons with Harmful Alcohol Consumption-II. Addiction 1993;88:791-804.

15. Rollnick S, Miller WR. What is motivational interviewing? Behav Cogn Psychother 1995;23:325-34.

16. Bandura A. Social foundations of thoughts and action: a social cognitive theory. Englewood Cliffs, New Jersey: Prentice-Hall, 1986.

17. Prochaska JO, DiClemente CC. Stages of change in the modification of problem behaviors. Prog Behav Modif 1992;28:183-218.

18. Pajala S. IKINÄ-opas (in Finnish). 183. The Finnish National Institute for Health and Welfare, 2018.

19. Gardner MMet al. Practical implementation of an exercise-based falls prevention programme. Age Ageing 2001;30:77-83.

20. Hauer K, Lamb SE, Jorstad EC, et al. Systematic review of definitions and methods of measuring falls in randomised controlled fall prevention trials. Age Ageing 2006;35:5-10.

21. Husu P, Suni J, Vähä-Ypyä H, et al. Objectively measured sedentary behavior and physical activity in a sample of Finnish adults: a crosssectional study. BMC Public Health 2016;16:y.

22. Hukkanen $\mathrm{H}$, Husu $\mathrm{P}$, Sievänen $\mathrm{H}$, et al. Aerobic physical activity assessed with accelerometer, diary, questionnaire, and interview in a Finnish population sample. Scand J Med Sci Sports 2018;28:2196-206.

23. Waller $\mathrm{K}$, Vähä-Ypyä $\mathrm{H}$, Törmäkangas $\mathrm{T}$, et al. Long-Term leisure-time physical activity and other health habits as predictors of objectively monitored late-life physical activity - a 40-year twin study. Sci Rep 2018;8.

24. Grimby G, Andrén E, Daving Y, et al. Dependence and perceived difficulty in daily activities in Community-Living stroke survivors 2 years after stroke. Stroke 1998;29:1843-9.
25. Rasinaho M, Hirvensalo M, Törmäkangas T, et al. Effect of physical activity counseling on physical activity of older people in Finland (ISRCTN 07330512). Health Promot Int 2012;27:463-74.

26. Podsiadlo D, Richardson S. The timed "Up \& Go": a test of basic functional mobility for frail elderly persons. J Am Geriatr Soc 1991;39:142-8.

27. Guralnik JM, Simonsick EM, Ferrucci L, et al. A short physical performance battery assessing lower extremity function: association with self-reported disability and prediction of mortality and nursing home admission. J Gerontol 1994;49:M85-M94.

28. Mathiowetz V, Kashman N, Volland G, et al. Grip and pinch strength: normative data for adults. Arch Phys Med Rehabil 1985;66:69-74.

29. Development of the World Health Organization WHOQOL-BREF quality of life assessment. The WHOQOL group. Psychol Med 1998;28:551-8

30. Kanis JA, Oden A, Johansson $\mathrm{H}$, et al. FRAX® and its applications to clinical practice. Bone 2009;44:734-43.

31. Sheikh JI, Yesavage JA, Brooks JO, et al. Proposed factor structure of the Geriatric Depression Scale. Int. Psychogeriatr. 1991:3:23-8

32. Powell LE, Myers AM. The Activities-specific Balance Confidence (ABC) scale. J Gerontol A Biol Sci Med Sci 1995;50A:M28-M34.

33. Huskisson EC. Measurement of pain. Lancet 1974;304:1127-31.

34. Miller-Davis C, Marden S, Leidy NK. The New York Heart Association classes and functional status: what are we really measuring? Heart Lung 2006;35:217-24.

35. Orthostatic test. Scand J Clin Lab Invest 1984;44:38-54.

36. Borg GAV. Psychophysical bases of perceived exertion. Med Sci Sports Exerc 1982;14:377???381-81.

37. Moyé LA. Multiple analyses in clinical trials: fundamentals for investigators. New York: Springer, 2003.

38. Rantanen T, Portegijs E, Viljanen A, et al. Individual and environmental factors underlying life space of older people-study protocol and design of a cohort study on life-space mobility in old age (LISPE). BMC Public Health 2012;12:1018.

39. Gray AM, Clarke PM, Wolstenholme JL, et al. Applied methods of cost-effectiveness analysis in healthcare. Oxford, New York: Oxford University Press, 2011

40. Muthén LK, Muthén BO. Mplus user's guide: statistical analysis with latent variables. 850. Los Angeles, CA: Muthén \& Muthén, 2009. 